Article

\title{
Dopamine Incorporated Forward Osmosis Membranes with High Structural Stability and Chlorine Resistance
}

\author{
Yi Wang ${ }^{1}$, Zhendong Fang ${ }^{1}$, Chaoxin Xie ${ }^{{ }^{*}}$, Shuaifei Zhao ${ }^{3}$, Derrick $\mathrm{Ng}^{2}$ and Zongli Xie ${ }^{2, *}$ \\ 1 Water Industry and Environment Engineering Technology Research Centre, 401311, Chongqing, China; \\ a86909304@163.com; \\ 2 CSIRO Manufacturing, Clayton, VIC 3168, Australia; zongli.xie@csiro.au \\ 3. Department of Environmental Sciences, Macquarie University, Sydney, NSW 2109, Australia \\ * Correspondence: a86909304@163.com; Tel.: +86-023-731001; zongli.xie@csiro.au; Tel.: +61-3-95452938;
}

\begin{abstract}
The degradation and detachment of the polyamide (PA) layer in thin-film composite (TFC) membranes due to chlorine based chemical cleaning and material difference of PA layer and substrate are two major bottlenecks of forward osmosis (FO) technology. In this study, a new type of FO membranes were prepared by controlling self-polymerization of dopamine (DA) in the aqueous phase and the reaction with trimesoyl chloride (TMC) during interfacial polymerization (IP) process. These membranes were characterized by attenuated total reflection Fourier transform infrared (ATR-FTIR), X-ray photoelectron spectroscopy (XPS), field-emission scanning electron microscopy (FESEM) and water contact angle measurements. The influence of synthesis parameters such as $\mathrm{pH}$ of the aqueous phase, reaction time, temperature, and monomer concentrations were systematically investigated. The optimized membrane showed enhanced structure stability in ethanol (7.1 times higher) and chlorine resistance (72.3 times higher) than the conventional Piperazine(PIP)/TMC membrane due to (poly-dopamine) PDA bio-adhesion and polyester groups in the membrane structure. In general, DA/TMC membranes could be an effective strategy to fabricate high-performance FO membranes with excellent structural stability and chlorine resistance.
\end{abstract}

Keywords: forward osmosis; thin-film composite; dopamine; interfacial polymerization; structural stability; chlorine resistance.

\section{Introduction}

In recent years, FO has attracted increasing interest in desalination ${ }^{1-3}$, wastewater treatment ${ }^{4-5}$, osmotic membrane bioreactors ${ }^{6-7}$, fertilizers ${ }^{8-9}$ and power generation ${ }^{10-11}$, as a potential low-energy and more environmentally friendly technology ${ }^{12}$. Forward osmosis (FO) is a natural process that drives water molecules across a semi-permeable membrane from the feed solution (with a low osmotic pressure) to the draw solution (with a high osmotic pressure). Thin-film composite polyamide (TFC-PA) membranes consisting of an active PA layer and a porous substrate layer have been widely investigated for FO due to their excellent separation capability, wide range $\mathrm{pH}$ tolerance and independently tailorable support layer and active layer properties ${ }^{13-16}$.

Generally, the thin active polyamide (PA) layer is obtained by interfacial polymerization over the support layer by two monomers with amino groups and acid chloride groups ${ }^{17}$. In order to meet the crosslinking and mechanical properties of the PA layer, at least one selected monomer must have more than two functional groups as well as an aromatic compound ${ }^{18}$. The concentration of the monomers, the reaction time and temperature of the interfacial polymerization can all affect the separation performance of the membrane ${ }^{15}$. However, if the conventional TFC membranes are used in severe conditions containing organic solvents like ethanol, the active layer could be easily detached from the substrate, since there is no strong linkage between the PA layer and porous substrate. So the 
membrane structural stability should be taken into serious consideration for more practical membrane separation applications. Apart from the membrane structural stability, membrane chlorine resistance is also a bottleneck for TFC-PA membrane applications. This is because membrane fouling is inevitable in the FO process, for example, in biological fouling it is the main cause of decreasing membrane performance ${ }^{5,13,19}$. Therefore, membrane cleaning with oxidizing agents to control membrane biofouling is also needed in FO applications. However, the antioxidant ability of the conventional PA layer is poor, and its polymer chain structure is easily degraded by active chlorine and consequently bring a significantly reduced membrane separation performance ${ }^{20-21}$. Hence, TFC-PA membranes with improved structural stability and chorine resistance are highly desirable.

In recent years, mussel-inspired dopamine (DA) or polydopamine (PDA) chemistry, has shown a new route for the fabrication of high-performance membranes. PDA with adhesive proteins have been used to improve fouling resistance of ultrafiltration (UF) ${ }^{22}$ and RO membranes ${ }^{23}$, by forming surface-adherent films onto either the selective layer or the support layer to increase hydrophilic property of membranes. Han et al.[16] first designed time-different PDA coated substrates for FO membrane synthesis and found that FO membrane based on $1 \mathrm{~h}$-PDA-coated substrate showed the best FO performance. Inspired by Han's work, Huang et al..$^{24}$ prepared three kinds of substrates (PDA coated on top, bottom and dual surface substrate, respectively) with PDA for $1 \mathrm{~h}$ before the formation of TFC membranes, observing a reduction of reverse solute flux for the PDA coated top surface membranes. Very recently, Guo et al. ${ }^{25}$ proved that only $0.5 \mathrm{~h}$ coating was enough for PDA deposition and modification on the PA active layer, which would enhance FO membrane selectivity as well as anti-fouling property.

Inspired by these works, DA was first explored as a sole monomer in aqueous phase to prepare thin film composite membranes by interfacial polymerization in FO membrane synthesis. The amine and phenol groups in DA, and the catechol groups of PDA in the aqueous phase ${ }^{26-27}$ can all react with acyl chloride groups of trimesoylchloride (TMC) in the organic phase to obtain a new type of thin film active layer for separation applications, which is strongly adhered to the top of the polysulfone substrate. At the same time, the novel active layer, with ester bonds formed by DA/TMC, is much more stable than the amide bonds of the PA layer when exposed to solutions with active chloride. State-of-the-art characterization techniques including attenuated total reflection Fourier transform infrared (ATR-FTIR), X-ray photoelectron spectroscopy (XPS) and field-emission scanning electron microscope (FESEM), were applied to characterize the as-prepared membranes. This study offers significant insights into developing high-performance FO membranes by exploring the relationship between the self-polymerization of dopamine in aqueous phase and FO performance.

\section{Materials and Methods}

\subsection{Materials}

Polysulfone (PSf, average molecular weight (MW) $22 \mathrm{kDa}$, Sigma-Aldrich, USA), polyvinylpyrrolidone (PVP, average MW $\sim 10 \mathrm{kDa}$, Sigma Aldrich, USA), 1-methyl-2-pyrrolidinone (ACI Labscan, Thailand), 1-methyl-2-pyrrolidinone (NMP, 99\%, RCI LABSCAN LIMITED, Thailand), 1, 3, 5-Benzenetricarbonyl chloride (TMC, Alfa Aesar ${ }^{\circledR}$, England, 98\%) were dispersed in hexane (Univar, Redmond, WA) and dopamine hydrochloride (International Laboratory, USA, 99\%). For membrane performance tests, Magnesium chloride $\left(\mathrm{MgCl}_{2}\right.$, ACS reagent) was dissolved in deionized water (DI) obtained from a Milli-Q ultra pure water purification system (Millipore, Billerica, MA). For membrane structural stability and chlorine resistance tests, ethanol (ACS, EMSURE®, Germany) and sodium hypochlorite ( $\mathrm{NaClO}$ ) solution (Chem Supply Pty Ltd, Australia) with $8-12.5 \%$ available chlorine were used, respectively.

\subsection{Preparation of polysulfone substrates}

The porous polysulfone membrane substrate was fabricated via the phase inversion method using a PSf-PVP mixed casting solution. To prepare the casting solution, a mixture containing $15 \mathrm{~g}$ 
PSf, $8 \mathrm{~g}$ PVP and $77 \mathrm{~g}$ NMP was magnetically stirred for $24 \mathrm{~h}$ and then left standing for approximately $12 \mathrm{~h}$ at room temperature for degassing ${ }^{28-29}$. The prepared casting solution was first cast onto a clean and smooth glass plate using a membrane casting knife (RK Print Coat Instruments Ltd., UK) with controlled wet film thickness $(195 \pm 10 \mu \mathrm{m})$, and then exposed to air for approximately $10 \mathrm{~s}$ before it immersion in a $60{ }^{\circ} \mathrm{C}$ DI water bath for $30 \mathrm{~min}$ to initiate the phase inversion process. Next, the membrane was removed from the water bath, thoroughly rinsed with Milli-Q water and then transferred into a $4{ }^{\circ} \mathrm{C}$ DI water bath for storage and later use.

\subsection{Preparation of thin film composite (TFC) membranes}

The TFC composite membranes were prepared via interfacial polymerization between the mixed-amine (DA and PDA) solution and TMC on the surface of the PSf substrates prepared as below. Specifically, a series of dopamine-containing solutions were prepared by mixing phosphate buffered solution (PBS) with different concentrations of dopamine $(0.10,0.30,0.50 \mathrm{wt} \%)$. The PSf substrate was first immersed in the prepared solution for $30 \mathrm{~min}$ to ensure complete soaking and appropriate self-polymerization of DA ${ }^{30}$. The excess solution was removed with an air-knife (Cincinnati, Ohio USA) within $30 \mathrm{~s}$. The substrate was then dipped into TMC solution $(0.15 \mathrm{wt} \%$ in hexane) for $60 \mathrm{~s}$, to initiate interfacial polymerization. Next, the substrate was removed from the hexane organic phase and set vertically for $2 \mathrm{~min}$ to evaporate excess organic solution. Afterwards, a dry curing process was performed by placing the as-prepared TFC membrane into an oven at $90{ }^{\circ} \mathrm{C}$ for $5 \mathrm{~min}$. Finally, the obtained composite membranes were washed and stored in $4{ }^{\circ} \mathrm{C}$ DI water until testing. The resultant membranes were denoted as $\mathrm{M}-1, \mathrm{M}-2$ and $\mathrm{M}-3$, containing $0.10,0.30$ and 0.50 $w t \%$ of DA in the aqueous phase, respectively. For comparison, a conventional TFC-PA membrane formed by piperazine/TMC (denoted as M-0) was also prepared as the control membrane.

\subsection{Membrane characterizations}

The morphology of the membrane surfaces was observed by FESEM (Merlin ZEISS GEMINI2) operating at $5 \mathrm{kV}$ and a working distance of $6.5-7.0 \mathrm{~mm}$. Surface chemistry of the active layers was determined by XPS (ESCALab220IXL, VG Scientific Company Ltd., England) with an Al/K $\alpha$ (hv = $1486.6 \mathrm{eV}$ ) anode mono X-ray source. Water contact angles were measured using a static sessile drop contact angle system (DataphysicsOCA20) by placing a $2 \mu \mathrm{L}$ droplet on a dried membrane surface. For each membrane, 10 measurements were performed. The functional groups of the membranes were analysed using ATR-FTIR spectroscopy instrument (Thermo Scientific Nicolet 6700).

\subsection{FO performance tests}

FO performance experiments were conducted using a lab-scale filtration unit with an effective membrane area of about $42.0 \mathrm{~cm}^{2}$. A commercial PTFE cell (CF042-FO, Sterlitech Co.) was used to hold the membrane, having two rectangular channels with dimensions of $92.1 \mathrm{~mm}$ in length, $45.7 \mathrm{~mm}$ in width and $2.3 \mathrm{~mm}$ in depth. Co-current cross-flow mode was selected and the flow velocities were $4.9 \mathrm{cms}^{-1}$ for both the feed and draw solutions. The temperatures of the feed and draw solutions were controlled at $20 \pm 0.5^{\circ} \mathrm{C} .1 \mathrm{M}$ of $\mathrm{MgCl}_{2}$ solution was used as the draw solution and a digital balance was connected a computer to monitor weight change of the permeated water at $30 \mathrm{~s}$ intervals (ML4002, METTLER TOLEDO). DI water was used as the feed solution and a conductivity meter (Con 110, Oakton Instruments) was used to monitor the conductivity change at $60 \mathrm{~s}$ intervals for calculating the reverse solute flux of the membrane. Membranes were tested under two different modes: active layer facing draw solution (AL-DS) mode, and active layer facing feed solution (ALFS) mode. Each test was conducted for $60 \mathrm{~min}$ in triplicate.

The water permeation flux $\left(\mathrm{J}_{\mathrm{w}}\right)\left(\mathrm{L} \mathrm{m}^{-2} \mathrm{~h}^{-1}, \mathrm{LMH}\right)$ was calculated by the following equation:

$\mathrm{Jw}=\frac{\Delta \mathrm{w}}{\mathrm{om}_{\mathrm{e}} \mathrm{t}}$

where $\Delta \mathrm{w}(\mathrm{kg})$ is the mass change of the draw solution over a period time $\mathrm{t}(\mathrm{h})$, $\mathrm{Q}$ is the density of water $\left(\mathrm{kg} \mathrm{L}^{-1}\right)$ and $\mathrm{me}$ is the effective membrane area $\left(\mathrm{m}^{2}\right)$. 
The reverse solute flux, (Js) $\left(\mathrm{g} \mathrm{m}^{-2} \mathrm{~h}^{-1}\right)$, salt leakage or reverse diffusion from the draw solution to the feed solution, is an important parameter in FO determined by the increase of the feed solution conductivity.

$$
\mathrm{Js}=\frac{\Delta \mathrm{CtVt}}{\mathrm{m}_{\mathrm{e}} \mathrm{t}}
$$

where $\Delta \mathrm{C}_{\mathrm{t}}$ and $\mathrm{V}_{\mathrm{t}}$ are the change of concentration and volume of the feed solution at the end of FO experiment, respectively.

The specific reverse solute flux $(\mathrm{g} / \mathrm{L})$, which means the ratio of reverse solute flux/water flux $(\mathrm{Js} / \mathrm{Jw})$ ratio, is also calculated to measure the selectivity of the resultant TFC membranes.

\subsection{Evaluation of structural stability and chlorine resistance}

The structural stability of membranes was studied by immersing the prepared TFC membranes in ethanol for a fixed period. Chlorine resistance of the as-prepared membranes was evaluated by exposing the membrane surfaces to $1000 \mathrm{ppm} \mathrm{NaClO}$ solution over different periods at room temperature. To maintain the constant concentration of $\mathrm{NaClO}$ during the experiment, the container of $\mathrm{NaClO}$ solution was kept in darkness and $\mathrm{NaClO}$ solution was replaced every $24 \mathrm{~h}$. Before the membrane testing, the $\mathrm{FO}$ membranes were removed from ethanol or $\mathrm{NaClO}$ and washed thoroughly with DI water for 3 times to avoid the residual chlorine and ethanol swelling during performance tests.

\section{Results and discussion}

\subsection{Membrane Surface Characterization}

\subsubsection{Membrane Surface Chemistry}

It is well known that TFC membrane possesses a dense and thin layer on top of the substrate, which is critical for high membrane water flux and salt rejection ${ }^{31}$. In this study, ATR-FTIR and XPS analysis were utilised to confirm the reaction between DA/PDA and TMC, and the formation of these thin layers. The ATR-FTIR spectra for PSf-substrate, M-1, M-2 and M-3 are presented Figure 1a. It is clear that, apart from the bands of the PSf substrates, the DA/TMC TFC series membranes showed enhanced peaks at $1660 \mathrm{~cm}^{-1}$ and additional peaks at $1750 \mathrm{~cm}^{-1}$, which are attributed to the $\mathrm{C}=\mathrm{O}$ stretching in amide bonds and $\mathrm{C}=\mathrm{O}$ breathing in ester bonds, respectively. These characteristic bands indicated that the DA/TMC thin film active layers were obtained successfully onto the top of the PSf substrates. Moreover, nitrogen was detected by XPS on the DA/TMC membrane surfaces (Figure 1b), which further confirmed the existence of the DA/PDA on the top of substrates since amine groups were the exclusive N source ${ }^{16}$. High-Resolution XPS spectrum C 1 s spectra of the M-2 surface is also shown in Figure 1c, the peaks at bonding energies of 285.2, 286.1, 287.4 and $288.2 \mathrm{eV}$ can be attributed to the $\mathrm{C}-\mathrm{C}, \mathrm{C}-\mathrm{N}$ and $\mathrm{C}-\mathrm{O}, \mathrm{O}=\mathrm{C}-\mathrm{N}$ and $\mathrm{O}=\mathrm{C}-\mathrm{N}$ functional groups, respectively. presence of 287.4 and $288.2 \mathrm{eV}$ peaks further confirmed the formation of ester and amide groups which are resulted from the reaction between the catechol of PDA (or phenolic hydroxyl and amine groups in DA) and acyl chloride in TMC monomers. Based on ATR-FTIR and XPS analysis results, the formation of additional PA layers on the top of PSf substrates has been successful.
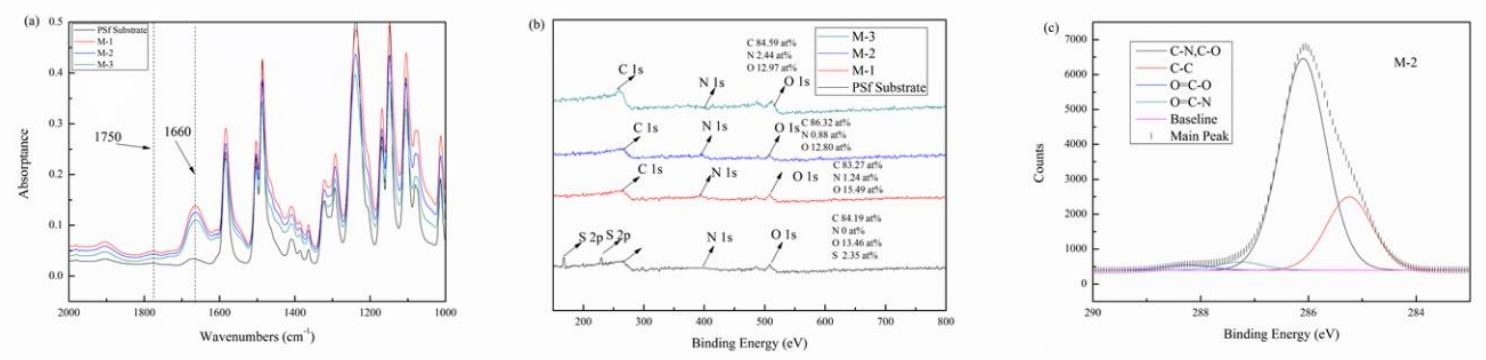
Figure 1. (a) ATR-FTIR spectra of PSf substrate, M-1, M-2 and M-3; (b) XPS spectra of the M-1, M-2 and M3 membrane surface and (c) High-Resolution XPS spectrum C 1s spectra of M-2 surface.

\subsubsection{Membrane Surface Morphology}

Figure 2 shows the SEM images of the as-prepared membranes. It is clear that the surface of the PSf substrate was smooth. As for the PIP/TMC conventional TFC-PA (M-0), a typical "valley and peak" structure was found, confirming the formation of polyamide, which is similar to Dong et al.'s work ${ }^{28}$. As for the DA/TMC membrane surface (M-1, M-2, and M-3), nodular structures on membrane surfaces were found in the SEM images. When the DA concentration was low $(0.1 \mathrm{wt} \%)$ in the aqueous phase, a relatively smooth surface with small and random specks could be found. As the DA concentration increased to $0.3 \mathrm{wt} \%(\mathrm{M}-2)$, the appearance of these specks became more uniform and frequent, resulting in a rougher surface these imperfections/pass ways could be beneficial for enhancing water molecules transfer. However, when the DA concentration was increased to $0.5 \mathrm{wt} \%$ (M-3), agglomeration was clearly observed, which may reduce the membrane rejection.
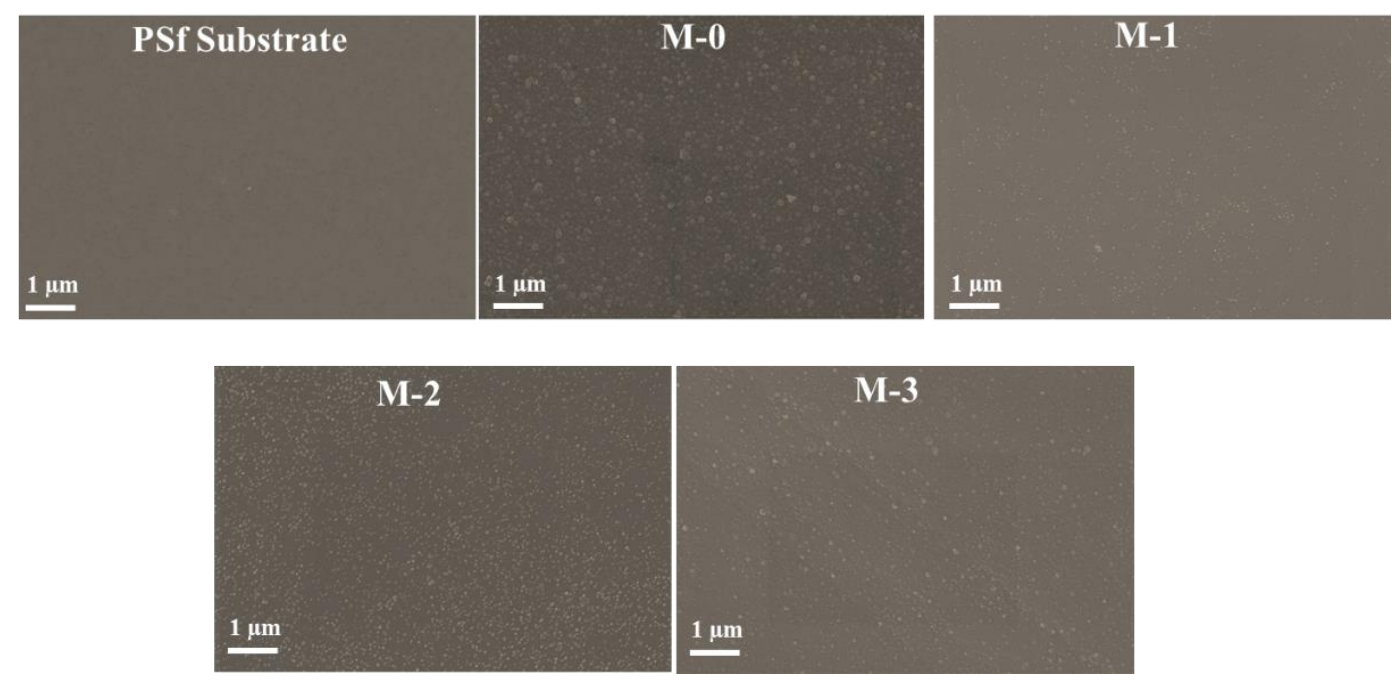

Figure 2. SEM images of PSf substrate, M-0, M-1, M-2 and M-3 membrane surface.

\subsubsection{Membrane Surface Hydrophilicity}

It is expected that the introduction of DA and PDA with abundant amine and hydroxyl groups would result in an increased surface hydrophilicity, which has been confirmed by the water contact angle analysis (Figure 3). Water contact angle is a classical measurement to test the wettability of membrane surfaces: the lower the water contact angle, the easier for water to wet the membrane surface and the higher the hydrophilicity. The water contact angle of the PSf substrate was relatively high at $75.3^{0}$. The water contact angle of the conventional membrane (M-0) was $51.0^{\circ}$, which is similar to Mohsen et al.'s work ${ }^{32}$. For the DA/TMC membranes with different DA concentrations, the water contact angles slightly decreased from $55.9^{\circ}$ to $50.2^{\circ}$ when the DA concentration in aqueous phase was increased from $0.1 \mathrm{wt} \%$ to $0.5 \mathrm{wt} \%$. These results indicate that the DA concentrations in the aqueous phase had slight positive influence on the membrane surface hydrophilicity.

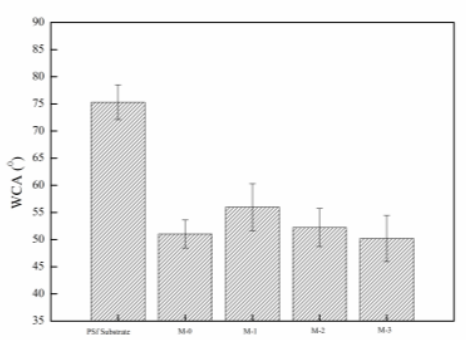


Figure 3. Water contact angles of PSf substrate, M-0, M-1, M-2 and M-3.

\subsection{Membrane FO Performance and Preparation Parameter Optimization}

Figure 4a shows the influence of DA concentration in the aqueous phase on FO performance of the $\mathrm{DA} / \mathrm{TMC}$ TFC membranes. The concentration of DA was fixed at $0.1,0.3$ and $0.5 \mathrm{wt} \%$ in the aqueous phase, respectively. The FO water flux increased from 3.22 to $6.55 \mathrm{LMH}$. Further increasing the DA concentration to $0.5 \%$ had little influence on the water flux. However, the specific reverse flux was almost doubled with high error bars. This is because high DA concentration would easily aggregate on the top of the membrane and thus some PDA pinnacles (see Figure 2) might randomly increase the FO water flux but at the price of salt rejection. Based on the above experimental results, the reaction temperature and reaction time of DA/TMC for interfacial polymerization were fixed at $25{ }^{\circ} \mathrm{C}$ and $120 \mathrm{~s}, 0.3 \mathrm{wt} \% \mathrm{DA}$ and $\mathrm{pH}$ value 7 in the aqueous phase, respectively as the optimized parameters. The formation DA/TMC of active layer was determined by not only the interfacial polymerization process, but also the self-polymerization of DA monomers. The $\mathrm{pH}$ value of the aqueous phase, IP reaction time, temperature and DA concentrations would directly affect these two processes, thus overall affecting membrane $\mathrm{FO}$ process. The influence of $\mathrm{pH}$ value in the aqueous phase on membrane $\mathrm{FO}$ performance was first studied for two reasons. First, according to Zhu's study, self-polymerization activity would be highly enhanced in alkaline solutions and reached the highest level at $\mathrm{pH} 8.5^{33}$. Another reason is that hydrochloric acid would be produced as a result of the IP reaction between amine and phenol groups in DA monomer and acyl chloride groups in TMC monomer. Thus $\mathrm{pH}$ value would certainly affect the processing of the positive reaction of the chemical equilibrium ${ }^{34}$.

Figure $4 \mathbf{b}$ shows the influence of $\mathrm{pH}$ value in aqueous phase on the FO performance of the DA/TMC TFC membranes. At the $\mathrm{pH}$ of 6.0, the FO water flux was relatively high (9.51 LMH) as well as the specific reverse solute flux $(1.62 \mathrm{~g} / \mathrm{L})$, which indicated that acidic environment had a negative impact on both self-polymerization and IP process. When the $\mathrm{pH}$ value was increased to 7 , the water flux sharply decreased to $6.55 \mathrm{LMH}$, and the specific reverse solute flux decreased even further to $0.4 \mathrm{~g} / \mathrm{L}$. When the $\mathrm{pH}$ value was further increased to 7.5 and 8.0, the FO water flux continuously decreased while the specific reverse solute flux slightly increased. This could be due to the process of DA self-polymerization was greatly enhanced at alkali solution environment ${ }^{35}$, thus, less amine and phenolic hydroxyl groups of DA could react with TMC in IP process. As a result, a low salt rejection and loose active layer was thus formed. From this, $\mathrm{pH} 7$ was chosen in the following experiments.

Figure 4c shows the influence of reaction time on the FO performance of the DA/TMC TFC membranes. When the IP reaction time was $10 \mathrm{~s}$, only a little amount of amine and phenol groups in DA monomer could react with TMC, as a result, the main component of the active layer was loose PDA, which is attributed to the high FO water flux and low salt rejection. When the reaction time increased from $60 \mathrm{~s}$ to $120 \mathrm{~s}$, FO water flux decreased from 8.32 to $6.55 \mathrm{LMH}$ and the specific reverse solute fluxes decreased from 1.37 to $0.4 \mathrm{~g} / \mathrm{L}$. However, further increase in the reaction time did not decrease the specific reverse solute flux obviously, but reduced the FO water flux. This might be attributed to the fact that too long IP reaction time increased the active layer crosslinking degree thus a relatively dense active layer was formed.

Figure $4 \mathrm{~d}$ shows the influence of reaction temperature on the FO performance of the DA/TMC TFC membranes. When the IP reaction temperature was $15^{\circ} \mathrm{C}$, a very high $\mathrm{FO}$ water flux $(9.08 \mathrm{LMH})$ and low salt selectivity $(0.65 \mathrm{~g} / \mathrm{L})$ was obtained. When the reaction temperature was increased from 25 to $55^{\circ} \mathrm{C}$, FO water flux decreased from 6.55 to $2.54 \mathrm{LMH}$ and the specific reverse solute fluxes gradually decreased from 0.40 to $0.32 \mathrm{~g} / \mathrm{L}$, respectively. This might be attributed to the fact that higher IP reaction temperature increased the reaction between DA and TMC. The obtained dense active layer lowered the FO water flux but maintained membrane selectivity. Based on the above experimental results, the reaction temperature and reaction time of DA/TMC for interfacial polymerization were fixed at $25^{\circ} \mathrm{C}$ and $120 \mathrm{~s}, 0.3 \mathrm{wt} \% \mathrm{DA}$ and $\mathrm{pH}$ value 7 in the aqueous phase, respectively as the optimized parameters. 

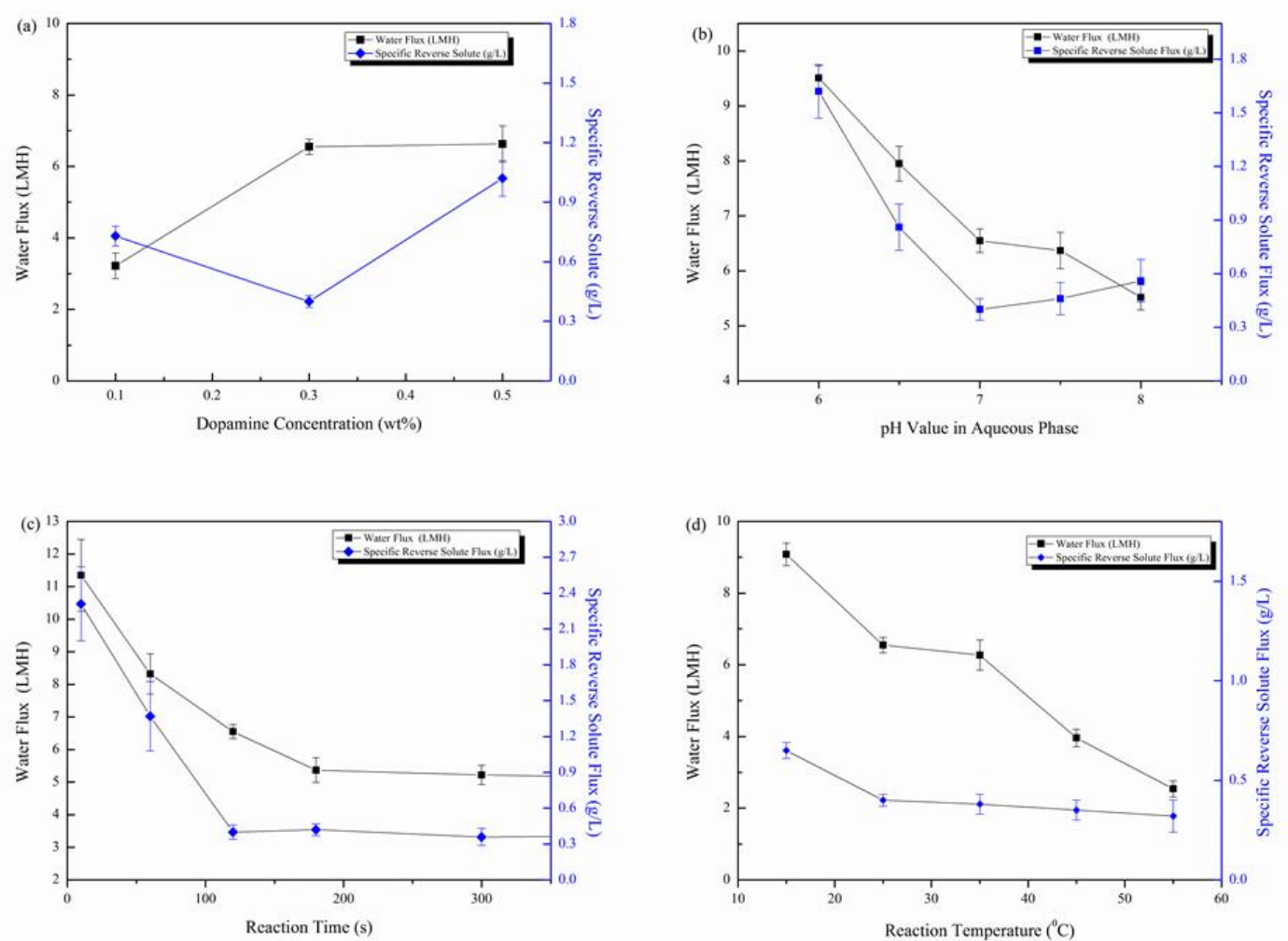

Figure 4. (a) The influence of DA concentration ( $0.1 \mathrm{wt} \%, 0.3 \mathrm{wt} \%$ and $0.5 \mathrm{wt} \%)$ in aqueous phase on the FO performance of the DA/TMC TFC membranes; $(\mathbf{b})$ The influence of $\mathrm{pH}$ value $(6,6.5,7,7.5$ and 8$)$ in aqueous phase on the FO performance of the DA/TMC TFC membranes; (c) The influence of reaction time (10 - 300 s) on the FO performance of the DA/TMC TFC membranes and (d) The influence of temperature $(15,25$, 35,45 and $55^{\circ} \mathrm{C}$ ) in aqueous phase on the FO performance of the DA/TMC TFC membranes.

\subsection{Membrane Structural Stability and Chloride Resistance}

In practical applications, membrane characteristics such as structural stability and chloride resistance are important for the lifespan of FO TFC membranes. In the first scenario, some feed solutions may contain organic solvents like ethanol which could massively swell the substrates and separation layers, which are generally comprised of two different materials, causing the detachment of active layers from the substrates, thus shortening membrane lifespan. In the second scenario, in order to control the bio-fouling in FO process, disinfectants with free chloride are widely used, which can inevitably cause the conformational changes of the PA chains, leading to drastic damage in FO membrane separation performance. In order to evaluate the structural stability and chloride resistance of the as-prepared membranes quantitatively, in our study, a doubled value increase in specific reverse solute flux was chosen as an upper limit, resembling when the PA layers were detached or degraded too dramatically to be acceptable in practical applications.

\subsubsection{Membrane Structural Stability}

The optimized DA/TMC membrane (M-2) and the conventional PIP/TMC membrane (M-0) were separately immersed in ethanol to examine the structural stability, their FO performances are shown in Figure 5. For M-2, its FO water flux increased from 6.55 LMH to 9.22 LMH after 228-hour ethanol immersion, and the specific reverse solute flux also increased to $0.515 \mathrm{~g} / \mathrm{L}$, it took 228 hours to double the initial specific reverse solute flux value. On the other hand, it only took $32 \mathrm{~h}$ for $\mathrm{M}-0$ to double its specific reverse solute flux. This enhancement of structural stability of M-2 ( 7.1 times stronger) might be ascribed to the strengthened bond formation between the active layer and the substrate under the selected optimal conditions. Namely, when the $\mathrm{pH}$ value of the 
aqueous phase was fixed at 7.0, small amounts of DA monomers were readily able to self-polymerize ${ }^{33}$ to form tightly adhesive PDA particles with plenty of $\pi-\pi$ and hydrogen-bonding interactions in between active layer and substrate ${ }^{36}$.
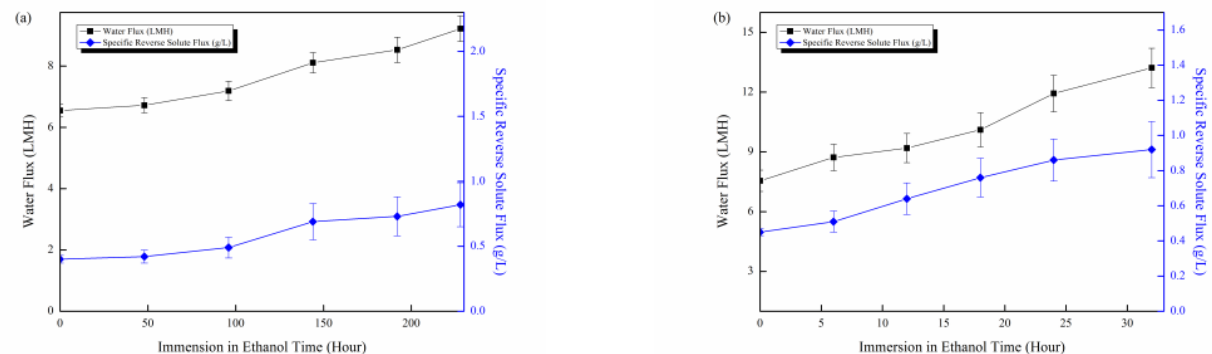

Figure 5. (a) The influence of ethanol immersion on the FO performance of M-2 membrane; (b) The influence of ethanol immersion on the FO performance of PIP/TMC conventional membrane. The FO performance was tested in active layer-feed solution (AL-FS) mode with $1.0 \mathrm{M} \mathrm{MgCl}_{2}$ as draw solution and DI water as the feed solution.

\subsubsection{Membrane Chlorine Resistance}

Chlorine-resistance studies were conducted for both DA/TMC (M-2) and PIP/TMC membranes (M-0) as shown in Figure 6. For M-2, a gradual increase of water flux and specific reverse solute were observed during the course of chlorine exposure, which lasted up to $12000 \mathrm{ppm} \mathrm{h}^{-1}$, before the specific reverse solute value was doubled. The ester bonds of M-2 were much more stable than amide bonds of PA membranes when exposed to chlorine. Therefore, a gradual increase of water flux due to the slow destruction of the surface inadequate crosslinking structure was observed. Meanwhile, due to a relatively complete active layer structure, the rise in reverse solute flux on top of the membrane was avoided. The water flux of the M-0 membrane was firstly increased like the trend for M-2 but eventually decreased to approximately $65 \%$ of the initial flux in $500 \mathrm{ppm} \mathrm{h}^{-1}$, while the specific reverse solute flux was finally increased from 0.45 to $3.05 \mathrm{~g} / \mathrm{L}$. This is because the amide bonds of the PA layers were vulnerable to free chlorine owing to their high electron density. At the beginning of the degradation of PA layer, the water molecules can easily pass through the membrane, however, because of the drastic degradation of the PA layer a highly increased reverse solute flux was observed, which caused an RO-like concentration polarization on top of the active layer thus decreasing water permeability and selectivity. Such significant failure of membrane separation performance may hinder the application of PIP/TMC TFC-PA membranes in wastewater treatment, which usually involves high bio-foulant components. The much slower destruction of the surface inadequate cross-linking structure as portrayed by M-2 was due to its more stable ester bonds, which are much stronger than amide bonds of PA membranes when exposed to chlorine, therefore showing far superior chlorine resistance characteristics (72.3 times enhancement). Additionally, due to its more complete active layer structure, the surging reverse solute flux on the top of membrane was avoided.
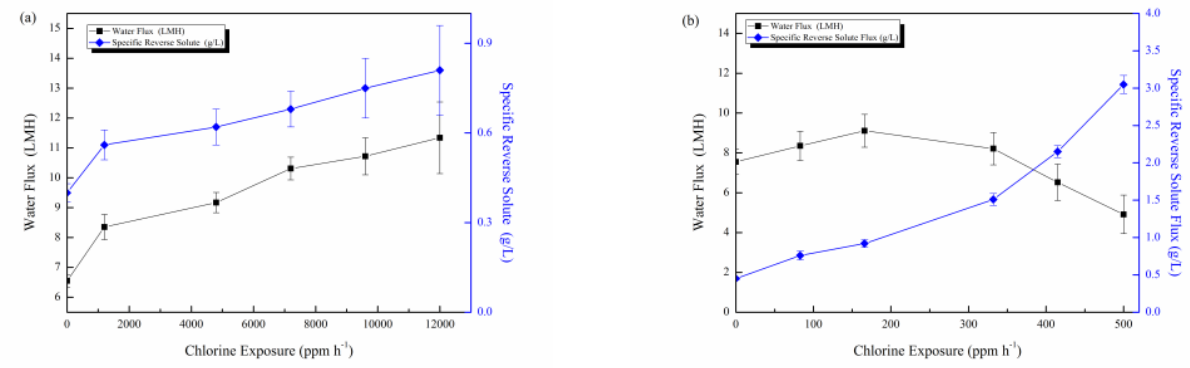
Figure 6. (a) The influence of chlorine immersion on the FO performance of M-2 membrane; (b) The influence of chlorine immersion on the FO performance of PIP/TMC conventional membrane. The FO performance was tested in active layer-feed solution (AL-FS) mode with $1.0 \mathrm{M} \mathrm{MgCl}_{2}$ as draw solution and DI water as the feed solution.

\section{Conclusions}

A new type of DA/TMC TFC membranes was prepared through the self-polymerization process of DA monomers and interfacial polymerization of DA and TMC monomers on PSf substrates. Uniform nodular structures were generated on the membrane surfaces of the novel membranes at a moderate DA concentration. The optimized parameters for this novel membrane synthesis process were obtained, including DA concentration $(0.3 \mathrm{wt} \%)$, reaction temperature $\left(25^{\circ} \mathrm{C}\right)$, reaction time $2 \mathrm{~min}$ and aqueous phase $\mathrm{pH}$ value 7 , respectively. The optimized membrane showed enhancement of 7.1 times more structurally stable and 72.3 times more chloride-resistant characteristics than the conventional PIP/TMC membrane due the PDA bio-adhesion and polyester groups in this novel membrane structure. The excellent structural stability and chloride resistance shown by this new type of membrane could facilitate wider applications of FO membranes in wastewater treatment.

Author Contributions: Conceptualization, Yi Wang and Zongli Xie; Methodology, Yi Wang; Investigation, Yi Wang and Derrick Ng; Resources, Chaoxin, Xie; Writing-Original Draft Preparation, Yi Wang; Writing-Review \& Editing, Yi Wang, Derrick Ng, Shuaifei Zhao and Zongli Xie; Supervision, Zongli Xie, Zhendong Fang and Chaoxin Xie; Funding Acquisition, Yi Wang, Chaoxin Xie and Zongli Xie.

Acknowledgments: The authors would like to acknowledge the financial support from CSIRO Manufacturing. Yi Wang acknowledges the collective support granted by the Graduate Student Research Innovation Project (CYB15128) in Chong Qing in 2015 and also thanks the scholarship from China Scholarship Council (201503170336). Special thanks are given to Dr Mark Greaves from CSIRO for the SEM training and imaging in this work.

Conflicts of Interest: The authors declare no conflict of interest.

\section{References}

1.Zhao, S.; Zou, L.; Mulcahy, D., Brackish water desalination by a hybrid forward osmosisnanofiltration system using divalent draw solute. Desalination 2012, 284, 175-181.

2.Choi, Y.-J.; Choi, J.-S.; Oh, H.-J.; Lee, S.; Yang, D. R.; Kim, J. H., Toward a combined system of forward osmosis and reverse osmosis for seawater desalination. Desalination 2009, 247 (1-3), 239-246.

3.Zhao, S.; Zou, L., Effects of working temperature on separation performance, membrane scaling and cleaning in forward osmosis desalination. Desalination 2011, 278 (1), 157-164. 4.Korenak, J.; Basu, S.; Balakrishnan, M.; Helix-Nielsen, C.; Petrinic, I., Forward Osmosis in Wastewater Treatment Processes. Acta Chim Slov 2017, 64 (1), 83-94.

5.Xue, W.; Yamamoto, K.; Tobino, T., Membrane fouling and long-term performance of seawater-driven forward osmosis for enrichment of nutrients in treated municipal wastewater. Journal of Membrane Science 2016, 499, 555-562.

6.Wang, X.; Zhao, Y.; Yuan, B.; Wang, Z.; Li, X.; Ren, Y., Comparison of biofouling mechanisms between cellulose triacetate (CTA) and thin-film composite (TFC) polyamide forward osmosis membranes in osmotic membrane bioreactors. Bioresource technology 2016, 202, 50-8. 
7.Wang, X.; Chang, V. W. C.; Tang, C. Y., Osmotic membrane bioreactor (OMBR) technology for wastewater treatment and reclamation: Advances, challenges, and prospects for the future. Journal of Membrane Science 2016, 504, 113-132.

8.Sahebi, S.; Phuntsho, S.; Eun Kim, J.; Hong, S.; Kyong Shon, H., Pressure assisted fertiliser drawn osmosis process to enhance final dilution of the fertiliser draw solution beyond osmotic equilibrium. Journal of Membrane Science 2015, 481, 63-72.

9.Phuntsho, S.; Shon, H. K.; Hong, S.; Lee, S.; Vigneswaran, S.; Kandasamy, J., Fertiliser drawn forward osmosis desalination: the concept, performance and limitations for fertigation. Reviews in Environmental Science and Bio/Technology 2011, 11 (2), 147-168.

10.Altaee, A.; Sharif, A.; Zaragoza, G.; Ismail, A. F., Evaluation of FO-RO and PRO-RO designs for power generation and seawater desalination using impaired water feeds. Desalination 2015, 368, 27-35.

11.He, W.; Wang, Y.; Shaheed, M. H., Energy and thermodynamic analysis of power generation using a natural salinity gradient based pressure retarded osmosis process. Desalination 2014, 350, 86-94.

12.McCutcheon, J. R.; McGinnis, R. L.; Elimelech, M., Desalination by ammonia-carbon dioxide forward osmosis: Influence of draw and feed solution concentrations on process performance. Journal of Membrane Science 2006, 278 (1-2), 114-123.

13.Faria, A. F.; Liu, C. H.; Xie, M.; Perreault, F.; Nghiem, L. D.; Ma, J.; Elimelech, M., Thin-film composite forward osmosis membranes functionalized with graphene oxide-silver nanocomposites for biofouling control. Journal of Membrane Science 2017, 525, 146-156.

14.Shen, L.; Xiong, S.; Wang, Y., Graphene oxide incorporated thin-film composite membranes for forward osmosis applications. Chemical Engineering Science 2016, 143, 194205.

15.Jia, Q.; Xu, Y.; Shen, J.; Yang, H.; Zhou, L., Effects of hydrophilic solvent and oxidation resistance post surface treatment on molecular structure and forward osmosis performance of polyamide thin-film composite (TFC) membranes. Applied Surface Science 2015, 356, 1105-1116.

16.Han, G.; Zhang, S.; Li, X.; Widjojo, N.; Chung, T.-S., Thin film composite forward osmosis membranes based on polydopamine modified polysulfone substrates with enhancements in both water flux and salt rejection. Chemical Engineering Science 2012, 80, 219-231.

17.Salter, R. J., Forward osmosis. Water Conditioning and Purification 2005, 48 (4).

18.Jianjun Qina, T.-S. C., Effect of dope flow rate on the morphology, separation performance, thermal and mechanical properties of ultrafiltration hollow fibre membranes. Journal of Membrane Science 1999, 157, 35-51.

19.Mi, B.; Elimelech, M., Organic fouling of forward osmosis membranes: Fouling reversibility and cleaning without chemical reagents. Journal of Membrane Science 2010, 348 (1-2), 337-345.

20.Sun, J.; Zhu, L.-P.; Wang, Z.-H.; Hu, F.; Zhang, P.-B.; Zhu, B.-K., Improved chlorine resistance of polyamide thin-film composite membranes with a terpolymer coating. Separation and Purification Technology 2016, 157, 112-119. 
21.Do, V. T.; Tang, C. Y.; Reinhard, M.; Leckie, J. O., Degradation of polyamide nanofiltration and reverse osmosis membranes by hypochlorite. Environmental science \& technology 2012, 46 (2), 852-9.

22.McCloskey, B. D.; Park, H. B.; Ju, H.; Rowe, B. W.; Miller, D. J.; Chun, B. J.; Kin, K.; Freeman, B. D., Influence of polydopamine deposition conditions on pure water flux and foulant adhesion resistance of reverse osmosis, ultrafiltration, and microfiltration membranes. Polymer 2010, 51 (15), 3472-3485.

23.Arena, J. T.; McCloskey, B.; Freeman, B. D.; McCutcheon, J. R., Surface modification of thin film composite membrane support layers with polydopamine: Enabling use of reverse osmosis membranes in pressure retarded osmosis. Journal of Membrane Science 2011, 375 (1-2), 55-62.

24.Huang, Y.; Jin, H.; Li, H.; Yu, P.; Luo, Y., Synthesis and characterization of a polyamide thin film composite membrane based on a polydopamine coated support layer for forward osmosis. RSC Adv. 2015, 5 (128), 106113-106121.

25.Guo, H.; Yao, Z.; Wang, J.; Yang, Z.; Ma, X.; Tang, C. Y., Polydopamine coating on a thin film composite forward osmosis membrane for enhanced mass transport and antifouling performance. Journal of Membrane Science 2018, 551, 234-242.

26.Zangmeister, R. A.; Morris, T. A.; Tarlov, M. J., Characterization of polydopamine thin films deposited at short times by autoxidation of dopamine. Langmuir : the ACS journal of surfaces and colloids 2013, 29 (27), 8619-28.

27.Hong, S.; Na, Y. S.; Choi, S.; Song, I. T.; Kim, W. Y.; Lee, H., Non-Covalent SelfAssembly and Covalent Polymerization Co-Contribute to Polydopamine Formation. Advanced Functional Materials 2012, 22 (22), 4711-4717.

28.Dong, L.-X.; Huang, X.-c.; Wang, Z.; Yang, Z.; Wang, X.-m.; Tang, C. Y., A thin-film nanocomposite nanofiltration membrane prepared on a support with in situ embedded zeolite nanoparticles. Separation and Purification Technology 2016, 166, 230-239.

29.Dong, L.-X.; Yang, H.-w.; Liu, S.-t.; Wang, X.-m.; Xie, Y. F., Fabrication and antibiofouling properties of alumina and zeolite nanoparticle embedded ultrafiltration membranes. Desalination 2015, 365, 70-78.

30.Zhao, J.; Su, Y.; He, X.; Zhao, X.; Li, Y.; Zhang, R.; Jiang, Z., Dopamine composite nanofiltration membranes prepared by self-polymerization and interfacial polymerization. Journal of Membrane Science 2014, 465, 41-48.

31.Freger, V., Nanoscale heterogeneity of polyamide membranes formed by interfacial polymerization. Langmuir : the ACS journal of surfaces and colloids 2003, 19 (11), 47914797.

32.Jahanshahi, M.; Rahimpour, A.; Peyravi, M., Developing thin film composite poly(piperazine-amide) and poly(vinyl-ethanol) nanofiltration membranes. Desalination 2010, 257 (1-3), 129-136.

33.Xi, Z.-Y.; Xu, Y.-Y.; Zhu, L.-P.; Wang, Y.; Zhu, B.-K., A facile method of surface modification for hydrophobic polymer membranes based on the adhesive behavior of poly(DOPA) and poly(dopamine). Journal of Membrane Science 2009, 327 (1-2), 244-253. 
34.Lee, K. P.; Arnot, T. C.; Mattia, D., A review of reverse osmosis membrane materials for desalination-Development to date and future potential. Journal of Membrane Science 2011, 370 (1-2), 1-22.

35.Haeshin Lee, S. M. D., William M. Miller, Phillip B. Messersmith, Mussel-Inspired Surface Chemistry for Multifunctional Coatings. Science 2007, 318 (5849).

36.Li, B.; Liu, W.; Jiang, Z.; Dong, X.; Wang, B.; Zhong, Y., Ultrathin and stable active layer of dense composite membrane enabled by poly(dopamine). Langmuir : the ACS journal of surfaces and colloids 2009, 25 (13), 7368-74. 